Check for updates

Cite this: Sustainable Energy Fuels, 2017, 1, 859

Received 6th February 2017

Accepted 30th March 2017

DOI: 10.1039/c7se00077d

rsc.li/sustainable-energy

\section{Copper light-catching electrodes for organic photovoltaics $\uparrow$}

\author{
H. Jessica Pereira, Oliver S. Hutter \$ G. Dinesha M. R. Dabera, Luke A. Rochford§ \\ and Ross. A. Hatton (D)*
}

\begin{abstract}
Optically thin copper films with a random array of sub-optical wavelength apertures couple strongly with light in the wavelength range $600-800 \mathrm{~nm}$ due to excitation of surface plasmonic resonances. Herein we show that this trapped light can be used to excite electronic transitions in a nearby strongly absorbing organic semiconductor before the plasmonic excitations dissipate their energy as heat into the metal. This energy transfer process is demonstrated using model small molecule and polymer photovoltaic devices (based on chloro-aluminium phthalocyanine: $\mathrm{C}_{60}$ and $\mathrm{PCE}-10: \mathrm{PC} \mathrm{C}_{70} \mathrm{BM}$ heterojunctions respectively) in conjunction with a nano-hole copper electrode formed by thermal annealing an optically thin $\mathrm{Cu}$ film supported on polyethylene terephthalate. The efficiency of this process is shown to be highest for wavelengths in the range 650-750 nm, which is part of the solar spectrum that is weakly absorbed by today's high performance organic photovoltaic devices, and so these findings demonstrate that this type of electrode could prove useful as a low cost light catching element in high performance organic photovoltaics.
\end{abstract}

\section{Introduction}

Organic photovoltaic (OPV) devices convert sunlight directly into electricity using thin films (70-200 $\mathrm{nm}$ ) of strongly absorbing organic semiconductors as the photoactive material, and offer the possibility of very low cost fabrication onto flexible substrates, combined with short energy payback times. ${ }^{\mathbf{1 , 2}}$ The compatibility with light-weight, flexible substrates opens the door to applications in building integration, ${ }^{2}$ transportation ${ }^{3}$ and wearable/smart electronics ${ }^{4-7}$ not accessible to conventional rigid flat plate photovoltaics. However, the low charge carrier mobility in organic semiconductors ${ }^{\mathbf{4}, 5}$ limits the thickness of the light harvesting layer to below that needed to absorb all useful photons from the incident solar spectrum, which in turn limits the power conversion efficiency $(\eta) \cdot{ }^{6,7}$ Over the past 8 years several ways to circumvent this constraint have been proposed based on using of $\mathrm{Au}$ and $\mathrm{Ag}$ nanoparticles to increase the photon-to-electron conversion efficiency by increasing light absorption in the photoactive layer. ${ }^{8}$ Noble metal nanoparticles couple strongly with visible light as a result of excitation of localised surface plasmon resonances (LSPRs), ${ }^{9}$ which are

Department of Chemistry, University of Warwick, CV4 7AL, UK. E-mail:Ross.Hatton@ warwick.ac.uk

$\dagger$ Electronic supplementary information (ESI) available. See DOI: 10.1039/c7se00077d

\$ Current Address: Stephenson Institute for Renewable Energy, University of Liverpool, L69 7ZF, UK.

$\S$ Current Address: Department of Chemistry, University of Birmingham, B15 2TT, UK. collective oscillations of the conduction band electrons confined to the interface between the metal and the surrounding dielectric medium, that occur when the particle dimensions are much smaller than the wavelength of the incident light. ${ }^{7}$ The very large absorption cross-section of noble metal nanoparticles ${ }^{\mathbf{1 0}}$ combined with the possibility of engineering the nanoparticle size and geometry to tune the LSPR frequency, offers the possibility for them to be used in OPVs to enhance light absorption across a broad range of wavelengths via two different mechanisms: (i) by efficient scattering of the incident light into the far-field, thereby increasing the path length through the photo-active layer; ${ }^{11}$ and/or (ii) by confining and concentrating the incident light as an intense near-field evanescent wave at the nanoparticle-dielectric interface (i.e. LSPR excitations), ${ }^{7,12}$ where the near-field enhancement decays exponentially into the surrounding dielectric over a range of several tens of nanometres. ${ }^{12}$ The latter approach requires that the light harvesting organic semiconductor is as close to the nanoparticle as possible, although not so close that the competing process of exciton quenching becomes significant. $^{12,13}$ In practice the optimal separation is of the order of 3$5 \mathrm{~nm} .{ }^{12,13}$ Due to the very large absorption coefficient in organic semiconductors used to harvest light in OPVs it is possible that this trapped light can be harnessed directly to excite electron transitions from highest occupied molecular orbital (HOMO) to lowest unoccupied molecular orbital (LUMO) in the adjacent organic semiconductor, before the surface plasmonic excitations dissipate their energy as heat due to ohmic losses in the metal. ${ }^{14}$ Unfortunately the dimensions of $\mathrm{Au}$ and $\mathrm{Ag}$ 
nanoparticles needed for efficient scattering and concentration of light is of the order of several tens of nanometres ${ }^{11}$ and so integration into OPV device architectures over large areas presents a significant challenge for scale-up. ${ }^{8}$

An alternative scalable approach that has received relatively little attention to date, but is well matched to OPV device architectures, is to trap the incident light ${ }^{6}$ as surface plasmonic excitations at the surface of a noble metal electrode having a random array of sub-optical wavelength apertures. ${ }^{7}$ Similar to metal nanoparticles, nano-holes in a noble metal film have a large absorption cross-section enabling strong coupling with plane wave incident light, ${ }^{15}$ whilst having the advantage of being confined to the plane of the electrode. Consequently, unlike metal nanoparticles, nano-apertures in a metal film electrode do not risk compromising the shunt resistance of a device with a very thin light harvesting layer, such as in an OPV. Also, unlike metal nanoparticles, metal nano-hole electrodes can support both propagating and localised plasmonic-excitations, which are strongly coupled. ${ }^{6,15,16}$ This approach to light trapping in OPVs was first proposed by Reilly et al. who used a $30 \mathrm{~nm}$ thick $\mathrm{Ag}$ electrode with a random distribution of circular apertures of the same radius formed by micro-sphere lithography. ${ }^{17}$ Stec et al. ${ }^{18}$ advanced that work in three important ways: (i) by developing a scalable method for the formation of a dense array of random apertures over large areas based on annealing optically thin ( $\sim 10 \mathrm{~nm}) \mathrm{Ag}$ films; (ii) by demonstrating that Au could be used in place of Ag; and (iii) by showing that OPV devices using random aperture nano-hole electrodes could actually outperform conventional ITO glass window electrodes in OPVs. Importantly the apertures formed by thermal annealing have a broad distribution of shapes and sizes which ensures a broad-band plasmonic response, which is well-matched to OPV applications. ${ }^{15}$ However, whilst $\mathrm{Ag}$ and $\mathrm{Au}$ are the preferred metals for this application, because of their high conductivity and relatively low optical losses ${ }^{14}$ the use of such high cost metals in OPVs inevitably erodes the cost advantage of OPVs over other types of thin film photovoltaics, and so there is a need to identify lower cost alternatives to these metals. $\mathrm{Cu}$ is attractive as an alternative to $\mathrm{Ag}$ owing to its abundance, high electrical conductivity and because it is $\sim 100^{\text {th }}$ of the cost of Ag. ${ }^{19}$ The optical losses in $\mathrm{Cu}$ due to intra-band transitions and free electron (Drude) losses are comparable to that of Au so it can support intense surface plasmon resonances in the visible region, ${ }^{20,21}$ although $\mathrm{Cu}$ has not received much attention as a plasmon-active material for photovoltaics due to its susceptibility to oxidation in air, which results in the formation of a surface oxide that damps surface plasmonic excitations. ${ }^{20,21}$ However, in recent years there has been considerable progress in the development of ultra-thin surface passivation layers for $\mathrm{Cu}$ which offer a means of addressing this problem, ${ }^{22,23}$ and so nano-structured $\mathrm{Cu}$ may yet prove useful for light trapping and concentration in OPVs. Herein we demonstrate for the first time a proof-of-principle that $\mathrm{Cu}$ electrodes with a random array of sub-optical wavelength apertures can be used to catch incident light and efficiently transfer that energy to an adjacent organic semiconductor in a model OPV device. Based on these findings we propose the optimal location for such an electrode to maximise its potential benefit in an OPV.

\section{Experimental}

\section{Substrate cleaning}

Substrate slides of the required dimension $(13 \times 13 \mathrm{~mm}$ for OPV devices and $26 \times 26 \mathrm{~mm}$ for electrode characterisation) were cut from a roll of PET (Mitsubishi, Hostaphan GN 125 4600A) and cleaned by rinsing consecutively in deionized water, 2-propanol (IPA) and acetone. The slides were then dried using a stream of nitrogen.

All electrode and device fabrication was carried out in a nitrogen filled glove box with a $\mathrm{O}_{2}$ level $<1 \mathrm{ppm}$ unless otherwise stated. Evaporation of metals and metal oxides was carried out with a CreaPhys Organic Molecular evaporator colocated in the same glove box. The thickness of deposited layers was monitored using a carefully calibrated quartz-crystal microbalance mounted adjacent to the substrate. All metals were thermally evaporated from tungsten boats. $\mathrm{MoO}_{3}$ was thermally evaporated using boron nitride crucibles. Chloroaluminium phthalocyanine (ClAlPc) and $\mathrm{C}_{60}$ were thermally evaporated from boron nitride crucibles. The working pressure of the evaporator was $<1 \times 10^{-5}$ mbar.

\section{Deposition of copper}

$\mathrm{Cu}$ was deposited by thermal evaporation of $\mathrm{Cu}$ pellets at a rate of 2.2-2.5 $\AA^{-1}$ to give a thickness of $11 \mathrm{~nm}$. Notably both sides of the PET sheet were not identical in surface roughness and so AFM imaging was performed in tapping mode using an Asylum Research MFP - 3D to determine the smooth side onto which $\mathrm{Cu}$ was deposited.

\section{Fabrication of apertures}

$\mathrm{Cu}$ films were annealed in a tube furnace at $175{ }^{\circ} \mathrm{C}$ under a pressure $\leq 5 \times 10^{-4}$ mbar for 10 minutes. Scanning electron microscopy (SEM) imaging was performed using ZEISS Supra SP55 with an accelerating voltage of typically $5-7 \mathrm{kV}$ to evaluate the distribution and coverage of apertures.

\section{Optical transparency measurement}

Far-field transmittance of metal films on PET was measured over the wavelength range $300-900 \mathrm{~nm}$ using $150 \mathrm{~mm}$ Spectralon ${ }^{\circledR}$ Integrating Sphere coupled to PerkinElmer ${ }^{\circledR}$ LAMBDA $^{\mathrm{TM}}$ high performance series of UV/Vis spectrometer. The incident beam passed through the plastic substrate first.

\section{Fabrication of OPV devices}

ClAlPc was purified by thermal gradient sublimation prior to deposition. All other chemicals were used without further purification. $\mathrm{MoO}_{3}\left(5 \mathrm{~nm}\right.$ at $0.3-0.5 \AA \mathrm{s}^{-1}$ ) was deposited on annealed copper thin films using thermal evaporation. For fabrication of devices with bilayer architecture ClalPc $(20 \mathrm{~nm})$ and $\mathrm{C}_{60}(40 \mathrm{~nm})$ were deposited by thermal evaporation at a rate of 0.9-1.2 $\AA^{-1}$ and 0.3-0.4 $\AA^{-1}$ respectively. For devices with bulk-heterojunction architecture, PCE10 (poly[4,8-bis(5-(2-ethylhexyl)thiophen-2-yl)benzo[1,2-b;4,5- $\left.b^{\prime}\right]$ dithiophene-2,6-diylalt-(4-(2-ethylhexyl)-3-fluorothieno[3,4-b]thiophene-)-2- 
carboxylate-2-6-diyl $])$ and $\mathrm{PC}_{70} \mathrm{BM}\left([6,6]\right.$-phenyl- $\mathrm{C}_{71}$-butyric acid methyl ester) were mixed in 2:3 mass ratio to make a $35 \mathrm{mg}$ $\mathrm{ml}^{-1}$ solution in chlorobenzene (97\%) and 1,8-diiodooctane (3\%) and deposited by spin coating from a static start at $4000 \mathrm{rpm}$ for 120 seconds, followed by a methanol treatment at $4000 \mathrm{rpm}$ for 30 seconds. These slides were then allowed to dry under vacuum overnight. Bathocuproine (BCP) (5 nm at 0.3-0.5 $\AA_{\mathrm{s}^{-1}}$ ) and $\mathrm{Al}$ (80 nm at 1.0-1.3 $\AA^{-1}$ ) were deposited by thermal evaporation. Al was deposited through a shadow mask to give an electrode area of $0.06 \mathrm{~cm}^{2}$.

\section{Characterization of OPV devices}

Current density-voltage (JV) testing was performed in the dark and under 1 sun simulated solar illumination using a Keithley 2400 source meter and a custom Labview interface using an ABET technologies Sun 2000 Solar Simulator. The intensity was set to $100 \mathrm{~mW} \mathrm{~cm} \mathrm{~cm}^{-2}$ over the AM1.5 solar spectrum. The light source was calibrated using a calibrated silicon diode. EQE measurements were done using a white light xenon arc lamp (Sciencetech SF150), monochromator (Photon Technology International), focussing and splitting lenses, current-voltage amplifier (Femto DHPCA-100), lock-in amplifier (Stanford Research SR830 DSP) and a custom Labview interface.

\section{Results and discussion}

$\mathrm{Cu}$ films with a thickness of $11 \mathrm{~nm}$ were deposited onto the transparent plastic substrate polyethylene terephthalate (PET) by thermal evaporation, forming uniform films with a sheet resistance of $\sim 11 \Omega \mathrm{sq}^{-1}$ (Fig. 1(a)). Thermal annealing of these films under high vacuum results in the formation of a random array of irregularly shaped sub-optical wavelength apertures, the number density and size being determined by the annealing time and temperature (Fig. 1(b)). The glass transition temperature and melting point of PET are $67-81{ }^{\circ} \mathrm{C}$ and $\sim 220{ }^{\circ} \mathrm{C}$ respectively ${ }^{24}$ and so annealing increases the crystallinity of the PET substrate, as is evident from the X-ray diffraction data in Fig. S2. $\dagger$ However, this change in crystallinity does not change the far-field transparency; Fig. $\mathrm{S} 3, \dagger$ and so the change in the optical properties of the $\mathrm{Cu}$ electrode upon annealing result from a change in the $\mathrm{Cu}$ film morphology. The optimal annealing conditions were determined by observing aperture formation as a function of annealing temperature using a heated stage mounted inside a scanning electron microscope (SEM) (ESI Fig. S1 $\dagger$ ). When the electrodes were heated in vacuum $\left(5 \times 10^{-4} \mathrm{mbar}\right)$ at $175{ }^{\circ} \mathrm{C}$ for 10 minutes, a dense random array of apertures with dimensions in the size range 50-100 nm and area coverage of $\sim 10 \%$ were formed. Thermal annealing was accompanied by an increase in sheet resistance of the films to $\sim 27 \Omega \mathrm{sq}^{-1}$ which can be attributed to the combined effects of the inclusion of apertures and increase in film surface roughness, both of which increase the scattering of electrons as they move in the plane of the metal film under the influence of the applied bias. Importantly, this increase in sheet resistance is not sufficient to significantly change the series resistance of the OPV devices fabricated as part of this study because the devices have a small area of $0.06 \mathrm{~cm}^{2}$.

The incorporation of apertures into the $\mathrm{Cu}$ film results in a large reduction in the far-field transparency across a broad wavelength range between $450-850 \mathrm{~nm}$, with the largest absolute reduction of $10 \%$ for wavelengths in the range $590-700 \mathrm{~nm}$. Light scattering measurements performed using an integrating sphere (Fig. 1(c)) confirmed that this reduction in transparency is not the result of far-field light scattering, which is consistent with the small size of the apertures compared to the wavelength of the incident light. Since the largest reduction in transparency occurs for wavelengths in the range $590-700 \mathrm{~nm}$ and peaks at $\sim 625 \mathrm{~nm}$, the reduction in transparency for wavelength $>600 \mathrm{~nm}$ can be attributed (primarily) to light absorption arising from excitation of surface plasmon resonances in the $\mathrm{Cu}$ film, since this wavelength range coincides with that over which $\mathrm{Cu}$ can support surface plasmon resonance excitations and the optical losses due to inter-band transitions and free electron losses are at their lowest. ${ }^{14}$ Finite-element modelling of the near-field intensity in $\mathrm{Cu}$ films with and without apertures of the size and shapes
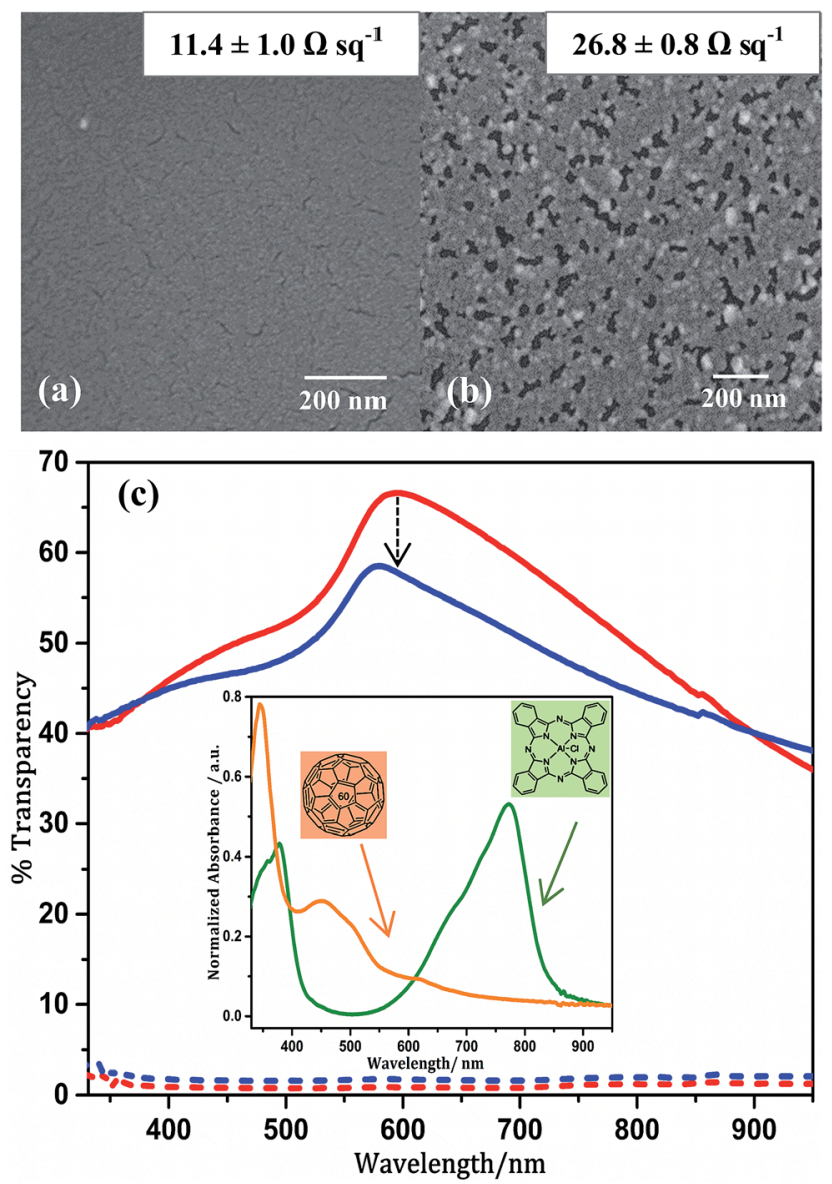

Fig. 1 SEM showing the morphology of Cu films (11 nm) before (a) and after (b) annealing at $175^{\circ} \mathrm{C}$ for $10 \mathrm{~min}$ at $5 \times 10^{-4} \mathrm{mbar}$; (c) optical transparency (solid lines) and far-field scattering (dashed lines) measurements of $11 \mathrm{~nm}$ Cu films with (blue) and without (red) nanoapertures inset: absorption spectra of CIAIPc $(20 \mathrm{~nm})$ and $\mathrm{C}_{60}(40 \mathrm{~nm})$ films - the same thickness used in the model OPV devices fabricated as part of this study. 

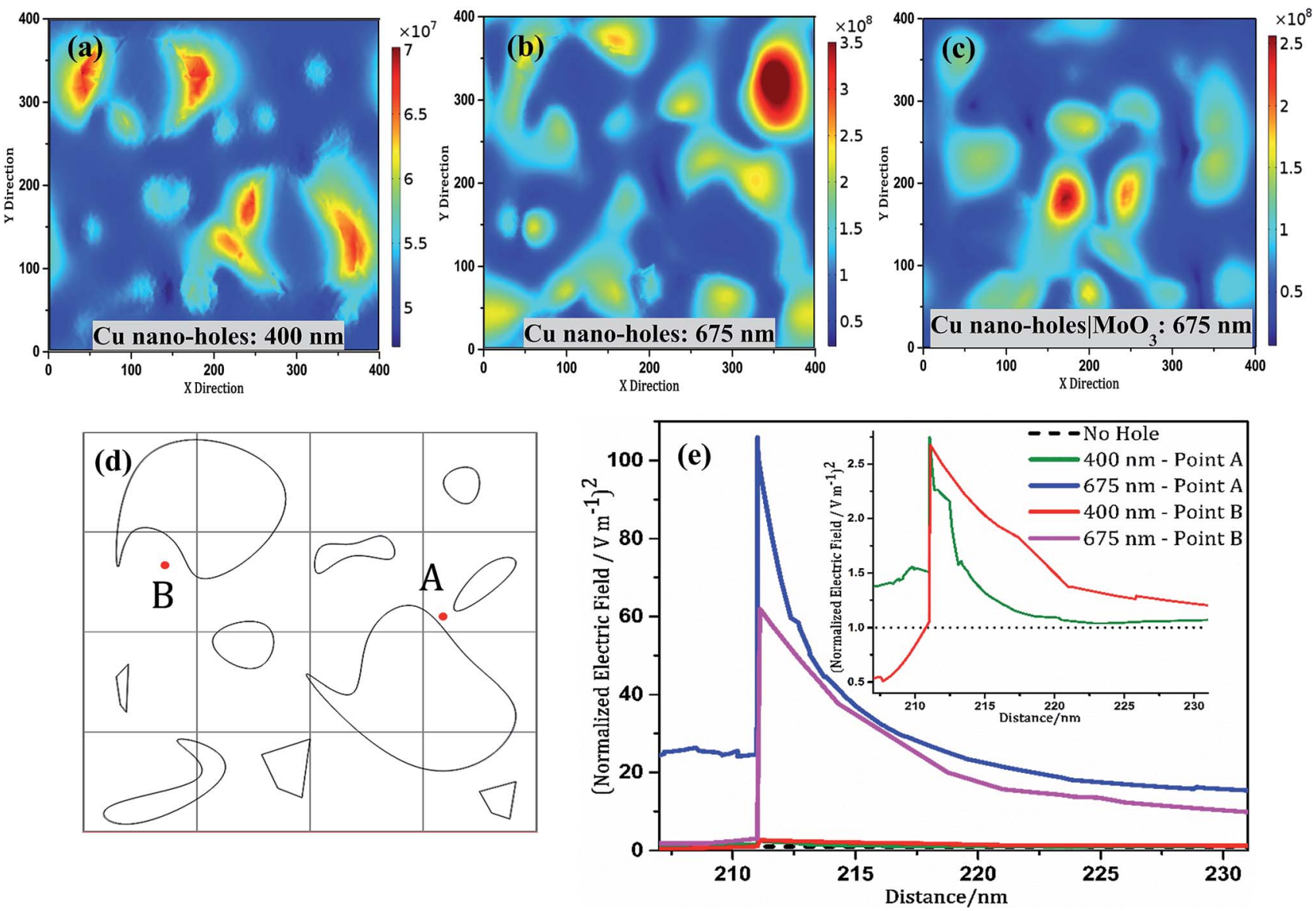

Fig. 2 Finite-element modelling of the near field $15 \mathrm{~nm}$ above the surface of an $11 \mathrm{~nm}$ thick Cu nano-hole electrode supported on PET, with a random arrangement of different shaped apertures (representative of that achieved experimentally) (d); optical field for $\lambda=400 \mathrm{~nm}$ (a) and $\lambda=$ $675 \mathrm{~nm}$ (b) plane wave incident light; (c) optical field $15 \mathrm{~nm}$ above the metal electrode for plane wave incident light of $\lambda=675 \mathrm{~nm}$, simulated for PET $(200 \mathrm{~nm}) / \mathrm{Cu}(11 \mathrm{~nm}) / \mathrm{MoO}_{3}(5 \mathrm{~nm}) / \mathrm{ClAlPc}(20 \mathrm{~nm})$; (e) normalized field strength as a function of distance from the surface of the Cu nanohole electrodes at points A and B (indicated in (d)) for plane wave incident PET $(200 \mathrm{~nm}) / \mathrm{Cu}(11 \mathrm{~nm})$.

formed by annealing $\mathrm{Cu}$ films corroborate the conclusion that light is trapped at the surface as a near-field evanescent wave (Fig. 2). The simulations also show that the intensity distribution across the electrode surface is highly heterogeneous, consistent with the complex interaction between plasmonic excitations associated with different features on the electrode surface. In principle these regions of intense near-field radiation can be used to excite electronic transitions from the HOMO to the LUMO in the organic semiconductor before the surface plasmons dissipate their energy as heat due to ohmic losses in the metal..$^{25}$ To explore this possibility, model OPVs based on a bilayer heterojunction of the small molecule donor ClAlPc and acceptor $\mathrm{C}_{60}$ (Fig. 1(c) inset) were fabricated using $\mathrm{Cu}$ electrodes with and without a random array of apertures shown in Fig. 1(a) and (b). This simple device architecture and the thickness of the organic semiconductor layers were specifically selected as a model structure to test the aforementioned hypothesis: ClAlPc was used because its intense Q-band ${ }^{26,27}$ is located between $600-850 \mathrm{~nm}$ (Fig. 1(c) - inset) which coincides with the range over which surface plasmon resonant excitations are most intense in the $\mathrm{Cu}$ nano-hole electrode. $\mathrm{MoO}_{3}$ was used as the hole-extraction layer because it is well-known to facilitate efficient extraction of holes from most donor type organic semiconductors for thicknesses in the range 3-20 nm and its thickness can be accurately controlled by thermal evaporation. ${ }^{28-30}$ The results of the finite-element modelling given (Fig. 2(c) and (e)) show that a $5 \mathrm{~nm}$ thick $\mathrm{MoO}_{3}$ layer is sufficiently thin for there to be significant spatial overlap between the ClAlPc layer and the near-field evanescent wave extending from the electrode surface, since the range of the trapped light extends to several tens of nanometres from the electrode surface.

The device current-voltage characteristics in the dark and under 1 sun illumination are shown in Fig. 3(a) and Table 1. It is evident that the short-circuit current density $\left(U_{\mathrm{sc}}\right)$ is comparable for devices using $\mathrm{Cu}$ electrodes with and without nano-holes, despite the lower far-field transparency of the $\mathrm{Cu}$ nano-hole electrode, particularly over the wavelength range between 600$800 \mathrm{~nm}$ which coincides with that over which ClAlPc most strongly absorbs (Fig. 1(c) inset). This finding is consistent with the hypothesis that the light trapped as surface plasmonic excitations in the $\mathrm{Cu}$ nano-hole electrode (which results in the reduced transparency for $\lambda>575 \mathrm{~nm}$ in the nano-hole electrode) is to a large extent coupled into the adjacent ClAlPc layer, since in the bilayer device architecture the ClAlPc is the only layer within 
range of the evanescent wave extending from the surface of the nano-hole electrode (Fig. 2(c) and (e)). This conclusion is corroborated by the difference in the shape of the external quantum efficiency (EQE) spectra (Fig. 3(b)) from which it is evident that photons are converted to electrons in the external circuit more efficiently for devices using the nano-hole electrode for $\lambda=600-800 \mathrm{~nm}$, which is the same range over which the farfield optical transparency is observed to be reduced. Indeed the EQE is increased by $\sim 25 \%$ over the wavelength range of $655-$ $715 \mathrm{~nm}$, which coincides with the wavelength range over which the optical losses in the $\mathrm{Cu}$ nano-hole electrode due to inter-band transitions and free electron damping are at a minimum (Fig. 3(c)) and so surface plasmon resonances will be most intense. Conversely for $\lambda<575 \mathrm{~nm}$ the EQE is reduced in line with the reduction in far-field transparency for devices using the Cu nano-hole electrode. Normalizing the difference in EQE to the transparency (\% T) (Fig. 3(c)) show that the onset of enhanced absorption actually occurs very close to the minimum in the optical losses for $\mathrm{Cu}$.

Interestingly, devices using a nano-hole electrode also consistently exhibit a higher $V_{\text {oc }}$ and FF (Fig. 3(a) and Table 1). It is evident from Fig. 3(a) that this difference stems from the earlier onset of dark current injection and increased current leakage at revere bias in devices using $\mathrm{Cu}$ electrodes without nano-holes. Since $\mathrm{Cu}$ is known to diffuse into evaporated $\mathrm{MoO}_{3-x}$ films at room temperature ${ }^{30,31}$ it is possible that the former stems from a difference in the energy level alignment at the electrodeClAlPc interface due to a variation in the extent of n-type doping of the $\mathrm{MoO}_{3-x}$ layer by $\mathrm{Cu}^{30}$ since the increase in crystallinity upon annealing would be expected to impart increased lattice stability, thereby reducing the thermodynamic drive for $\mathrm{Cu}$ diffusion into the neighbouring $\mathrm{MoO}_{3-x}$ layer. To test this hypothesis the crystallinity of the $\mathrm{Cu}$ electrodes before and after thermal annealing was probed using grazing angle X-ray diffraction films, and the work function of $\mathrm{Cu} / \mathrm{MoO}_{3-x}(5 \mathrm{~nm})$ bilayer films were measured using the contact potential difference method. It is evident from the X-ray diffraction pattern (ESI Fig. S2 $\dagger$ ) that the crystallinity is increased upon annealing because the intensity of the peak corresponding to reflection from the $\mathrm{Cu}$ (111) plane is more intense after annealing. The work function of the electrode $/ \mathrm{MoO}_{3-x}$ bilayer is also significantly lower for the case in which the $\mathrm{Cu}$ electrode was not annealed; typical values were $\sim 5.7 \mathrm{eV}$ and $\sim 5.1 \mathrm{eV}$ for $\mathrm{Cu}$ electrodes with and without nano-holes respectively. Importantly, whilst this difference complicates the interpretation of device $J_{\mathrm{sc}}$, it cannot explain the change in the shape of the EQE spectrum.

To test the generality of the findings, OPVs were also fabricated using a bulk-heterojunction based on the narrow band gap polymer PCE10 in conjunction with [6,6]-phenyl- $\mathrm{C}_{71}$-butyric acid methyl ester (PCE10:PC $\left.\mathrm{P}_{70} \mathrm{BM}\right)(100 \mathrm{~nm})$ in place of ClAlPc: $\mathrm{C}_{60}$. Importantly in this device structure a smaller proportion of the total photoactive layer thickness is in the region of intense nearfield near to the $\mathrm{Cu}$ electrode because the PCE10: $\mathrm{PC}_{70} \mathrm{BM}$ is $30 \%$ thicker than the ClAlPc: $\mathrm{C}_{60}$ bilayer, and unlike the ClAlPc: $\mathrm{C}_{60}$ the PCE10:PC ${ }_{70} \mathrm{BM}$ absorbs strongly over a broad range of wavelengths, including in the range $450-600 \mathrm{~nm}$ where the $\mathrm{Cu}$ nanohole electrode is not plasmon-active. Consequently, the $J_{\mathrm{sc}}$ in
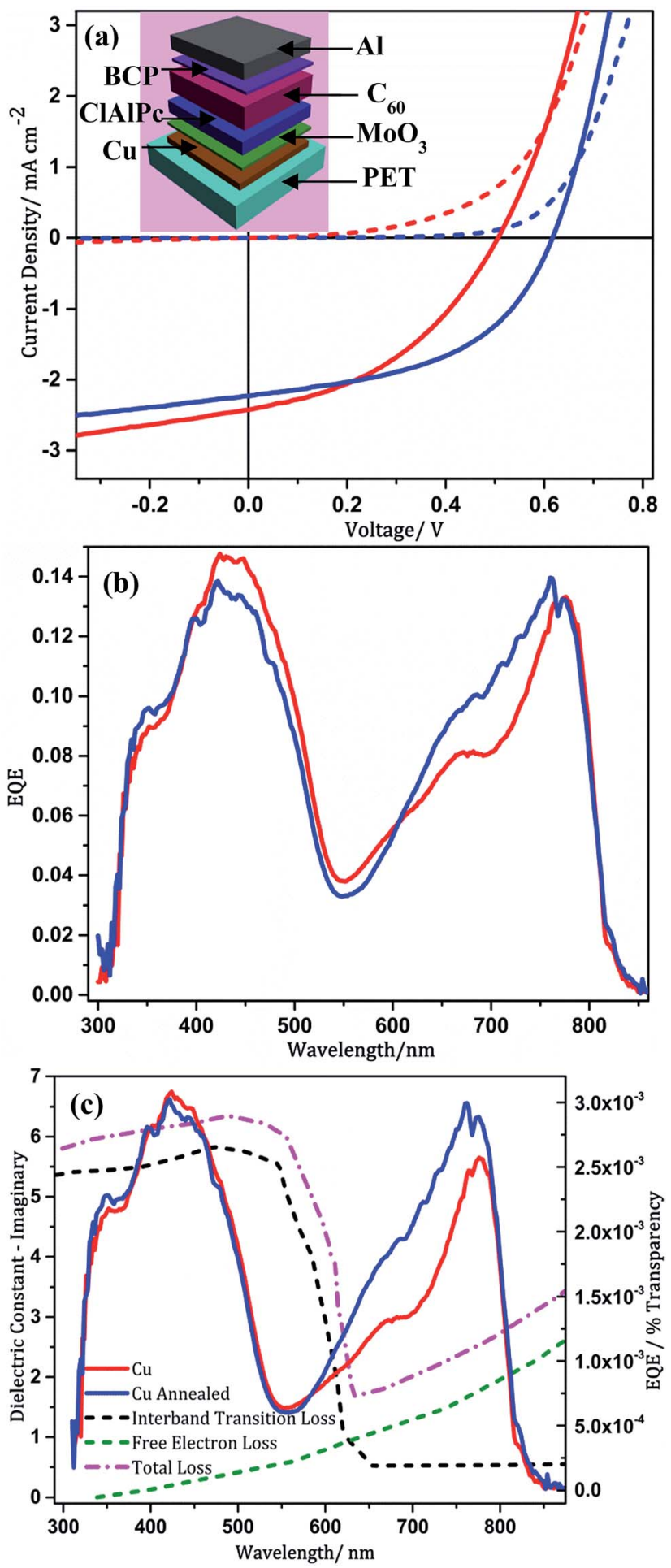

Fig. 3 (a) Representative current density-voltage characteristics in the dark and under one sun simulated solar illumination $(100 \mathrm{~mW}$ $\mathrm{cm}^{-2}$; AM 1.5G). Inset: schematic representation of the device architecture; (b) EQE for annealed (blue) and non-annealed (red) $\mathrm{Cu}$ electrodes for OPVs with the structure: $\mathrm{Cu}(11 \mathrm{~nm}) / \mathrm{MoO}_{3}(5 \mathrm{~nm}) /$ CIAIPC $(20 \mathrm{~nm}) / \mathrm{C}_{60}(40 \mathrm{~nm}) / \mathrm{BCP}(5 \mathrm{~nm}) / \mathrm{Al}(80 \mathrm{~nm}) ;$ (c) EQE/\% T vs. wavelength plot and imaginary component of the dielectric constant of $\mathrm{Cu}^{14}$ indicating inter-band transition losses and free electron losses. 
Table 1 Summary of current density-voltage characteristics under one sun simulated solar illumination for devices with the architecture; Cu (11 $\mathrm{nm}) / \mathrm{MoO}_{3}(5 \mathrm{~nm}) / \mathrm{ClAIPC}(20 \mathrm{~nm}) / \mathrm{C}_{60}(40 \mathrm{~nm}) / \mathrm{BCP}(5 \mathrm{~nm}) / \mathrm{Al}(80 \mathrm{~nm})$ with and without nano-holes in the Cu electrode

\begin{tabular}{|c|c|c|c|c|c|}
\hline Electrode & $\begin{array}{l}\text { Champion } \\
J_{\mathrm{sc}} / \mathrm{mA} \mathrm{cm}^{-2}\end{array}$ & $J_{\mathrm{sc}} / \mathrm{mA} \mathrm{cm}^{-2} \pm 1 \mathrm{SD}$ & $V_{\mathrm{oc}} / \mathrm{V} \pm 1 \mathrm{SD}$ & $\mathrm{FF} \pm 1 \mathrm{SD}$ & $\% \mathrm{PCE} \pm 1 \mathrm{SD}$ \\
\hline Non-annealed (without nano-holes) & 2.71 & $2.53 \pm 0.09$ & $0.48 \pm 0.03$ & $0.40 \pm 0.02$ & $0.49 \pm 0.04$ \\
\hline Annealed (with nano-holes) & 2.65 & $2.44 \pm 0.11$ & $0.60 \pm 0.02$ & $0.49 \pm 0.01$ & $0.72 \pm 0.05$ \\
\hline
\end{tabular}

Table 2 Summary of current density-voltage characteristics of devices with the architecture; $\mathrm{Cu}(11 \mathrm{~nm}) / \mathrm{MoO} 3(5 \mathrm{and} 15 \mathrm{~nm}) / \mathrm{PCE} 10-\mathrm{PC} 70 \mathrm{BM}$ $(100 \mathrm{~nm}) / \mathrm{BCP}(5 \mathrm{~nm}) / \mathrm{Al}(80 \mathrm{~nm})$ tested under 1 sun simulated illumination

\begin{tabular}{lllll}
\hline Electrode & $\begin{array}{l}\text { Thickness } \\
\text { of } \mathrm{MoO}_{3} / \mathrm{nm}\end{array}$ & $J_{\mathrm{sc}} / \mathrm{mA} \mathrm{cm}^{-2} \pm 1 \mathrm{SD}$ & $V_{\text {oc }} / \mathrm{V} \pm 1 \mathrm{SD}$ & $\mathrm{FF} \pm 1 \mathrm{SD}$ \\
\hline Non-annealed & 5 & $7.08 \pm 0.16$ & $0.65 \pm 0.04$ & $0.56 \pm 0.02$ \\
Annealed & 5 & $6.16 \pm 0.60$ & $0.72 \pm 0.02$ & $0.56 \pm 0.03$ \\
Non-annealed & 15 & $6.21 \pm 0.43$ & $0.71 \pm 0.01$ & $0.57 \pm 0.03$ \\
Annealed & 15 & $4.75 \pm 0.36$ & $0.74 \pm 0.01$ & $0.53 \pm 0.03$ \\
\hline
\end{tabular}


Fig. 4 Representative (a) current density-voltage characteristics in the dark and under 1 sun simulated illumination for OPVs with the structure: $\mathrm{Cu}(11 \mathrm{~nm}) / \mathrm{MoO}_{3}(5 \mathrm{~nm}) / \mathrm{PCE} 10-\mathrm{PC}_{70} \mathrm{BM}(100 \mathrm{~nm}) / \mathrm{BCP}(5$ $\mathrm{nm}) / \mathrm{Al}(80 \mathrm{~nm}) ;($ b) $E Q E / \% T$ vs. wavelength plot and imaginary component of the dielectric constant of $\mathrm{Cu}^{14}$ indicating inter-band transition losses and free electron losses. devices using nano-hole electrodes is lower than that without (Table 2). However, the EQE in devices using the nano-hole electrode is still highest in that part of the spectrum where the electrode is least transparent, which corresponds to region over which $\mathrm{Cu}$ supports surface plasmonic excitations. It is also evident from Fig. 4(b) that the onset of plasmon enhanced absorption occurs when the optical losses are a minimum and at the same wavelength as seen in the ClAlPc/C 60 OPVs; 590-600 nm (Fig. 3(c)).

To confirm the near-field nature of the light trapping mechanism, the thickness of the $\mathrm{MoO}_{3-x}$ layer was increased from 5 to $15 \mathrm{~nm}$. The key device performance parameters are summarised in Table 2. It is evident from Table 2 that the effect of increasing the $\mathrm{MoO}_{3-x}$ layer thickness from 5 to $15 \mathrm{~nm}$ is much more pronounced for the device with the nano-hole electrode, which is consistent with the exponential decay of the near-field intensity into the photoactive layer (Fig. 2(e)).

\section{Conclusions}

Collectively the findings reported herein provide compelling evidence that light trapped as surface plasmon-resonant excitations in an optically thin $\mathrm{Cu}$ electrode with a random array of sub-optical wavelength apertures can be efficiently coupled into an adjacent strongly absorbing organic semiconductor layer before the competing process of thermalisation can occur. This study has focused on demonstration of proof-of-principle in model OPVs, rather than optimisation of device performance. Based on the findings presented it may prove better to locate an electrode of this type at the back of the device so that the shorter wavelength photons can be substantially absorbed in the organic semiconductor before reaching the $\mathrm{Cu}$ electrode, since optical losses in $\mathrm{Cu}$ for $\lambda<600 \mathrm{~nm}$ are considerable.

\section{Acknowledgements}

The authors would like to thank the United Kingdom Engineering and Physical Sciences Research Council (EPSRC) for 
funding (Grant number: EP/N009096/1) and the University of Warwick for the award of a Chancellor's International Scholarship to H. Jessica Pereira.

\section{References}

1 B. Kippelen and J.-L. Bredas, Energy Environ. Sci, 2009, 2, 251-261.

2 B. P. Jelle, C. Breivik and H. Drolsum Røkenes, Sol. Energy Mater. Sol. Cells, 2012, 100, 69-96.

3 X. Fan, F. Wang, Z. Chu, L. Chen, C. Zhang and D. Zou, Appl. Phys. Lett., 2007, 90, 1-4.

4 W. U. Huynh, J. J. Dittmer, a P. Alivisatos and P. Alivisatos, Science, 2002, 295, 2425-2427.

5 P. Friederich, V. Meded, A. Poschlad, T. Neumann, V. Rodin, V. Stehr, F. Symalla, D. Danilov, G. Lüdemann, R. F. Fink, I. Kondov, F. von Wrochem and W. Wenzel, Adv. Funct. Mater., 2016, 5757-5763.

6 Z. Tang, W. Tress and O. Inganäs, Mater. Today, 2014, 17, 389-396.

7 H. A. Atwater and A. Polman, Nat. Mater., 2010, 9, 865.

8 Y. H. Jang, Y. J. Jang, S. Kim, L. N. Quan, K. Chung and D. H. Kim, Chem. Rev., 2016, 116, 14982-15034.

9 D. U. Karatay, M. Salvador, K. Yao, A. K. Y. Jen and D. S. Ginger, Appl. Phys. Lett., 2014, 105, 033304.

$10 \mathrm{M}$. Kuno, Introductory nanoscience: physical and chemical concepts, Garland Science, 2012.

11 X. Li, W. C. H. Choy, L. Huo, F. Xie, W. E. I. Sha, B. Ding, X. Guo, Y. Li, J. Hou, J. You and Y. Yang, Adv. Mater., 2012, 24, 3046-3052.

12 B. Niesen, B. P. Rand, P. Van Dorpe, D. Cheyns, H. Shen, B. Maes and P. Heremans, J. Phys. Chem. C, 2012, 116, 24206-24214.

13 W. P. Goh, E. L. Williams, R. Bin Yang, W. S. Koh, S. Mhaisalkar and Z. E. Ooi, ACS Appl. Mater. Interfaces, 2016, 8, 2464-2469.
14 P. R. West, S. Ishii, G. V. Naik, N. K. Emani, V. M. Shalaev and A. Boltasseva, Laser Photonics Rev., 2010, 4, 795-808.

15 T. H. Park, N. Mirin, J. B. Lassiter, C. L. Nehl, N. J. Halas and P. Nordlander, ACS Nano, 2008, 2, 25-32.

16 A. Uddin and X. Yang, J. Nanosci. Nanotechnol., 2014, 14, 1099-1119.

17 T. Reilly, J. van de Lagemaat, R. Tenent, A. Morfa and K. Rowlen, Appl. Phys. Lett., 2008, 92, 243304.

18 H. M. Stec and R. A. Hatton, Adv. Energy Mater., 2013, 3, 193199.

19 O. S. Hutter and R. A. Hatton, Adv. Mater., 2015, 27, 326-331. 20 G. H. Chan, J. Zhao, E. M. Hicks, G. C. Schatz and R. P. Van Duyne, Nano Lett., 2007, 7, 1947-1952.

21 C. Gong and M. S. Leite, ACS Photonics, 2016, 3, 507-513.

22 O. S. Hutter, H. M. Stec and R. A. Hatton, Adv. Mater., 2013, 25, 284-288.

23 M. Grouchko, A. Kamyshny and S. Magdassi, J. Mater. Chem., 2009, 19, 3057.

24 B. Demirel, A. Yaraş and H. Elçiçek, Suleyman Demirel Univ. Fen Bilimleri Enst. Derg., 2011, 13, 26-35.

25 N. N. Lal, H. Zhou, M. Hawkeye, J. K. Sinha, P. N. Bartlett, G. A. J. Amaratunga and J. J. Baumberg, Phys. Rev. B: Condens. Matter Mater. Phys., 2012, 85, 245318.

26 C. C. Leznoff and A. B. P. Lever, Phthalocyanines: properties and applications, 1989.

27 K. M. Kadish, K. M. Smith and R. Guilard, The porphyrin handbook, Academic Press, 2000.

28 M. Kröger, S. Hamwi, J. Meyer, T. Riedl, W. Kowalsky and A. Kahn, Appl. Phys. Lett., 2009, 95, 10-13.

29 R. T. White, E. S. Thibau and Z.-H. Lu, Sci. Rep., 2016, 6, 21109.

30 M. T. Greiner, L. Chai, M. G. Helander, W. M. Tang and Z. H. Lu, Adv. Funct. Mater., 2013, 23, 215-226.

31 D.-T. Nguyen, S. Vedraine, L. Cattin, P. Torchio, M. Morsli, F. Flory and J. C. Bernéde, J. Appl. Phys., 2012, 112, 63505. 